\title{
An innovative method for accelerating vaccine formulation development and manufacturing process: Using prefabricated vaccine base (standardized mixtures of vaccine excipients)
}

Author: Mulugeta Berhanu, Ethiopian Agricultural Transformation Agency

E-mail:mulugetach100@gmail.com; Tel: +251921433836

\begin{abstract}
Vaccine base can be defined as a standardized mixtures of vaccine excipients such as adjuvants emulsifier, preservatives, stabilizer, surfactant, buffers and etc. Vaccine bases are substances to which antigen (s) is/are added to form the final product (vaccine) which can provide protection against infectious disease. Prefabricated vaccine base can be used to develop vaccines in an easy and fast way. With vaccine base we do not need to formulate vaccines from scratch. Just we incorporate antigen of interest in vaccine base and vaccine is completed. Thus, premade vaccine base can be used to accelerate vaccine formulation development during global pandemics and epidemics. Using prefabricated vaccine base for vaccine development against various infectious diseases is an innovative method for accelerating deployment of existing and novel vaccines. Prefabrication of vaccine base is a new preparedness strategy for the future pandemics.
\end{abstract}

Key words: Accelerating vaccine formulation development, Antigen of interest, Prefabricated vaccine base, Vaccine, Vaccine Excipients 


\section{Introduction}

Innovations in process development and manufacturing are unavoidable to enable release of existing and novel vaccines and their delivery where and when they are mostly needed [1]. The vaccine industry is challenged to develop high-quality products at decreased cost and within shortened timelines. In return, shortening process development times will accelerate overall vaccine product development timelines and rapidly deliver safe and high-quality products to a global market [3]. Prefabricated vaccine base can be used to shorten vaccine formulation development and manufacturing timelines.

The idea of using prefabricated vaccine base for vaccine development against various infectious diseases is new and innovative. Prefabricated vaccine base is crucial for rapid development of existing and future vaccines. Prefabrication of vaccine base is a new preparedness strategy for the future pandemics. Vaccine base manufacturers and vaccine developers should work together to speed up vaccine development and manufacturing process.

The common vaccine excipients to be used in vaccine base formulation are adjuvants, emulsifier, preservatives, stabilizer, surfactant, buffers and etc. Adjuvants are immunostimulatory molecules that enhances immune response to vaccine antigens [2]. These molecules are the basic components of vaccine base to which antigen (s) is/are added to form vaccine which can provide protection against infectious disease. The excipients in vaccine bases for different vaccines vary depending on the vaccine type or antigen (s) in the vaccine. This means, vaccine bases for different vaccines may contain different adjuvants, emulsifier, preservatives, stabilizer, surfactant and buffers. 


\section{Vaccine Base Components}

Vaccine base is a standardized mixtures of vaccine excipients. Vaccine bases for different vaccines may contain different adjuvants, emulsifier, preservatives, stabilizer, surfactant and buffers (see table-1).

\begin{tabular}{|l|l|l|}
\hline No & Vaccine Base Components & Examples \\
\hline $\mathbf{1}$ & Adjuvants & $\begin{array}{l}\text { Aluminium salts (for many vaccines), MF-59 (for fluad } \\
\text { vaccine and others), AS03 (used in various vaccine } \\
\text { products), CpG 1018 (for heplisav-b® vaccine) }\end{array}$ \\
\hline $\mathbf{2}$ & Emulsifiers & Polysorbate 80, Sorbitan trioleate, Sodium citrate \\
\hline $\mathbf{4}$ & Stabilizers & $\begin{array}{l}\text { Thiomersal (commonly used preservative), 2- } \\
\text { phenoxyethanol. }\end{array}$ \\
\hline $\mathbf{5}$ & Surfactants & $\begin{array}{l}\text { Gelatin (for diptheria-tetanus-acellular pertussis vaccine, } \\
\text { flu vaccine and etc), MgCl } \text { (for OPV), MgSO } \text { (for } \\
\text { measles), lactose-sorbitol, lactose, sucrose, glycine, } \\
\text { monosodium glutamate, human or bovine (cow) serum } \\
\text { albumin }\end{array}$ \\
\hline $\mathbf{6}$ & Buffers & $\begin{array}{l}\text { Amphiphilic molecules --- Natural or synthetic origin, } \\
\text { non-ionic, cationic or amphoteric with a formula weight } \\
\text { between 600 and 4000 g/mol. }\end{array}$ \\
\hline
\end{tabular}

Table-1. Vaccine base components 


\section{Conclusions}

Vaccine base is the combination of vaccine excipients such as adjuvants, emulsifier, preservatives, stabilizer, surfactant, buffers and etc. Using prefabricated vaccine base is a new method of accelerating vaccine formulation development.

\section{References}

1. Anissa Boumlic-Courtade (2017): Accelerating vaccine process development and manufacturing: Innovative approaches and challenges. $21^{\text {st }}$ World Congress and Exhibition on Vaccines, Vaccination and Immunization November 09-10, 2017 Vienna, Austria.

2. Berhanu, M. (2021, July 30). Vaccine containing immunologic adjuvants with a wide range of activity to provide protection against COVID-19. https://doi.org/10.31730/osf.io/es9cf

3. Tony D'Amore and Yan-ping Yang (2019): Advances and Challenges in Vaccine Development and Manufacture. 